Supporting Information

Indolylarylsulfones as HIV-1 Non-Nucleoside Reverse Transcriptase Inhibitors: New Cyclic Substituents at the Indole-2-carboxamide

Giuseppe La Regina, Antonio Coluccia, Andrea Brancale, Francesco Piscitelli, Valerio Gatti, Giovanni Maga, Alberta Samuele, Christophe Pannecouque, Dominique Schols, Jan Balzarini, Ettore Novellino, and Romano Silvestri

\title{
TOC Graphic
}

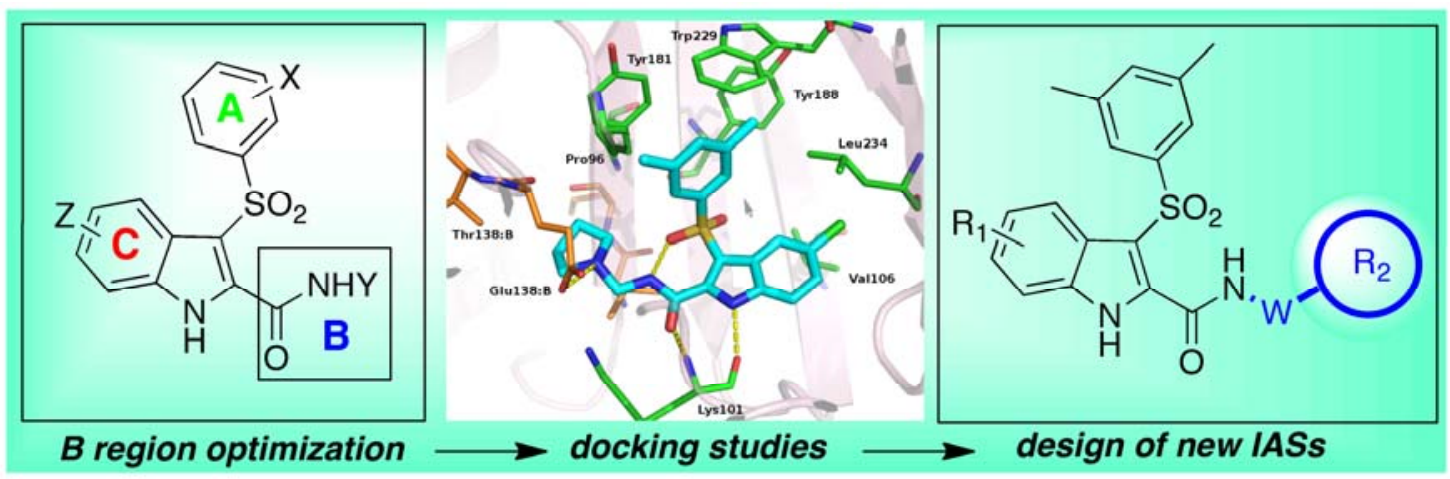

\section{Contents}

1) Preparative and spectroscopic data of derivatives 14, 16, 18, 20 and 22.

2) Preparative and spectroscopic data of derivatives 15, 17, 19, 21 and 23.

3) Preparative and spectroscopic data of derivatives 25, 26, 29, 30, 32-34, 36-38, 40-42 and 44-46.

4) Preparative and spectroscopic data of derivatives 28, 31, 35, 39, 43 and 47.

5) Figure 1S. Binding conformation of 12 into the NNBS of the HIV-1 L100I mutated RT.

6) Figure 2S. Binding conformation of 12 into the NNBS of the HIV-1 K103N mutated RT.

7) Figure 3S. Binding conformation of 12 into the NNBS of the HIV-1 Y181C mutated RT.

8) Table 1S. Anti-HIV-1 Activity, Cytotoxicity, Selectivity Index and Relative Factor of Compounds 20, 24-26, 28, 36, 40 and 44 against Mutant HIV-1 Strains in CEM Cell Cultures.

9) Table 2S. Inhibitory Effects of New IASs 12-47 and Reference Compound $\mathbf{4}$ on the Proliferation of Murine Leukemia Cells (L1210) and Human T-Lymphocyte Cells (Molt4/C8).

10) Table 3S. Elemental Analyses of derivatives 12-47 and 49. 
1) Preparative and spectroscopic data of derivatives 14, 16, 18, 20 and 22.

3-[(3,5-Dimethylphenyl)sulfonyl]-5-nitro- $N$-(pyrrolidin-1-ylmethyl)-1H-indole-2-

carboxamide (14). Was synthesized as 12 using 49. Yield $27 \%$, yellow solid, mp $256-259{ }^{\circ} \mathrm{C} .{ }^{1} \mathrm{H}$ NMR (DMSO-d $\left.)_{6}\right): \delta 1.71-1.75(\mathrm{~m}, 4 \mathrm{H}), 2.29(\mathrm{~s}, 6 \mathrm{H}), 2.73-2.78(\mathrm{~m}, 4 \mathrm{H}), 4.37(\mathrm{~d}, J=6.2 \mathrm{~Hz}, 2 \mathrm{H})$, $7.24(\mathrm{~s}, 1 \mathrm{H}), 7.62(\mathrm{~s}, 2 \mathrm{H}), 7.67(\mathrm{~d}, J=9.0 \mathrm{~Hz}, 1 \mathrm{H}), 8.11(\mathrm{dd}, J=2.2$ and $9.0 \mathrm{~Hz}, 1 \mathrm{H}), 8.84(\mathrm{~d}, J=2.3$ $\mathrm{Hz}, 1 \mathrm{H}), 9.27$ (br s, $1 \mathrm{H}$, disappeared on treatment with $\left.\mathrm{D}_{2} \mathrm{O}\right), 13.46 \mathrm{ppm}(\mathrm{br} \mathrm{s}, 1 \mathrm{H}$, disappeared on treatment with $\left.\mathrm{D}_{2} \mathrm{O}\right)$. IR: $v$ 1642, 3175, $3231 \mathrm{~cm}^{-1}$. Anal. $\left(\mathrm{C}_{22} \mathrm{H}_{24} \mathrm{~N}_{4} \mathrm{O}_{5} \mathrm{~S}(456.51)\right) \mathrm{C}, \mathrm{H}, \mathrm{N}, \mathrm{S}$.

5-Chloro-3-[(3,5-dimethylphenyl)sulfonyl]- $N$-(piperidin-1-ylmethyl)-1H-indole-2-

carboxamide (16). Was synthesized as 12 using piperidine. Yield $50 \%$, white solid, $\mathrm{mp} 172-174{ }^{\circ} \mathrm{C}$. ${ }^{1} \mathrm{H}$ NMR (DMSO-d $\left.d_{6}\right): \delta 1.35-1.37(\mathrm{~m}, 2 \mathrm{H}), 1.50-1.51(\mathrm{~m}, 4 \mathrm{H}), 2.31(\mathrm{~s}, 6 \mathrm{H}), 2.54-2.57(\mathrm{~m}, 4 \mathrm{H}), 4.21$ $(\mathrm{d}, J=5.9 \mathrm{~Hz}, 2 \mathrm{H}), 7.26(\mathrm{~s}, 1 \mathrm{H}), 7.34(\mathrm{dd}, J=1.9$ and $8.8 \mathrm{~Hz}, 1 \mathrm{H}), 7.55(\mathrm{~d}, J=8.1 \mathrm{~Hz}, 1 \mathrm{H}), 7.62(\mathrm{~s}$, 2H), $7.93(\mathrm{~d}, J=2.0 \mathrm{~Hz}, 1 \mathrm{H}), 9.19$ (br s, $1 \mathrm{H}$, disappeared on treatment with $\left.\mathrm{D}_{2} \mathrm{O}\right), 13.47 \mathrm{ppm}$ (br s, $1 \mathrm{H}$, disappeared on treatment with $\left.\mathrm{D}_{2} \mathrm{O}\right)$. IR: $v 1634,3189 \mathrm{~cm}^{-1}$. Anal. $\left(\mathrm{C}_{23} \mathrm{H}_{26} \mathrm{ClN}_{3} \mathrm{O}_{3} \mathrm{~S}(459.99)\right) \mathrm{C}$, $\mathrm{H}, \mathrm{Cl}, \mathrm{N}, \mathrm{S}$.

3-[(3,5-Dimethylphenyl)sulfonyl]-5-nitro- $N$-(piperidin-1-ylmethyl)-1H-indole-2-

carboxamide (18). Was synthesized as 12 using 49 and piperidine. Yield 41\%, white solid, mp 188$191{ }^{\circ} \mathrm{C} .{ }^{1} \mathrm{H}$ NMR (DMSO- $\left.d_{6}\right): \delta 1.37$ (s, 2H), 1.48-151 (m, 4H), $2.30(\mathrm{~s}, 6 \mathrm{H}), 2.56-2.60(\mathrm{~m}, 4 \mathrm{H}), 4.22$ $(\mathrm{d}, J=6.0 \mathrm{~Hz}, 2 \mathrm{H}), 7.26(\mathrm{~s}, 1 \mathrm{H}), 7.63(\mathrm{~s}, 2 \mathrm{H}), 7.69(\mathrm{~d}, J=9.1 \mathrm{~Hz}, 1 \mathrm{H}), 8.15(\mathrm{dd}, J=2.1$ and $9.2 \mathrm{~Hz}$, $1 \mathrm{H}), 8.83(\mathrm{~d}, J=2.2 \mathrm{~Hz}, 1 \mathrm{H}), 9.19$ (br s, $1 \mathrm{H}$, disappeared on treatment with $\left.\mathrm{D}_{2} \mathrm{O}\right), 13.42 \mathrm{ppm}(\mathrm{br} \mathrm{s}$, $1 \mathrm{H}$, disappeared on treatment with $\left.\mathrm{D}_{2} \mathrm{O}\right)$. IR: $v 1638,3161,3280 \mathrm{~cm}^{-1}$. Anal. $\left(\mathrm{C}_{23} \mathrm{H}_{26} \mathrm{~N}_{4} \mathrm{O}_{5} \mathrm{~S}(470.54)\right)$ $\mathrm{C}, \mathrm{H}, \mathrm{N}, \mathrm{S}$.

5-Chloro-3-[(3,5-dimethylphenyl)sulfonyl]- $N$-(morpholinomethyl)-1H-indole-2-

carboxamide (20). Was synthesized as 12 using morpholine. Yield 57\%, white solid, mp 209-211 ${ }^{\circ} \mathrm{C} .{ }^{1} \mathrm{H}$ NMR (DMSO- $\left.d_{6}\right): \delta 2.31(\mathrm{~s}, 6 \mathrm{H}), 2.61(\mathrm{t}, J=4.6 \mathrm{~Hz}, 4 \mathrm{H}), 3.60(\mathrm{t}, J=4.4 \mathrm{~Hz}, 4 \mathrm{H}), 4.22(\mathrm{~d}, J$ $=6.1 \mathrm{~Hz}, 2 \mathrm{H}), 7.26(\mathrm{~s}, 1 \mathrm{H}), 7.34(\mathrm{dd}, J=2.0$ and $8.8 \mathrm{~Hz}, 1 \mathrm{H}), 7.54(\mathrm{~d}, J=8.7 \mathrm{~Hz}, 1 \mathrm{H}), 7.65(\mathrm{~s}, 2 \mathrm{H})$, $7.92(\mathrm{~d}, J=2.1 \mathrm{~Hz}, 1 \mathrm{H}), 9.25\left(\mathrm{br} \mathrm{s}, 1 \mathrm{H}\right.$, disappeared on treatment with $\left.\mathrm{D}_{2} \mathrm{O}\right), 13.03 \mathrm{ppm}(\mathrm{br} \mathrm{s}, 1 \mathrm{H}$, disappeared on treatment with $\left.\mathrm{D}_{2} \mathrm{O}\right)$. IR: $v 1650,3201 \mathrm{~cm}^{-1}$. Anal. $\left(\mathrm{C}_{22} \mathrm{H}_{24} \mathrm{ClN}_{3} \mathrm{O}_{4} \mathrm{~S}(461.96)\right) \mathrm{C}, \mathrm{H}$, $\mathrm{Cl}, \mathrm{N}, \mathrm{S}$.

3-[(3,5-Dimethylphenyl)sulfonyl]- $N$-(morpholinomethyl)-5-nitro- $1 \mathrm{H}$-indole-2-carboxamide (22). Was synthesized as 12 using 49 and morpholine. Yield $41 \%$, yellow solid, mp $183-185{ }^{\circ} \mathrm{C} .{ }^{1} \mathrm{H}$ NMR (DMSO-d $\left.)_{6}\right): \delta 2.30(\mathrm{~s}, 6 \mathrm{H}), 2.58-2.62(\mathrm{~m}, 4 \mathrm{H}), 3.57-3.62(\mathrm{~m}, 4 \mathrm{H}), 4.21(\mathrm{~d}, J=6.0 \mathrm{~Hz}, 2 \mathrm{H})$, $7.27(\mathrm{~s}, 1 \mathrm{H}), 7.65(\mathrm{~s}, 2 \mathrm{H}), 7.70(\mathrm{~d}, J=9.0 \mathrm{~Hz}, 1 \mathrm{H}), 8.17(\mathrm{dd}, J=2.3$ and $9.1 \mathrm{~Hz}, 1 \mathrm{H}), 8.82(\mathrm{~d}, J=2.7$ $\mathrm{Hz}, 1 \mathrm{H}), 9.26$ (br s, $1 \mathrm{H}$, disappeared on treatment with $\left.\mathrm{D}_{2} \mathrm{O}\right), 13.48 \mathrm{ppm}(\mathrm{br} \mathrm{s}, 1 \mathrm{H}$, disappeared on treatment with $\left.\mathrm{D}_{2} \mathrm{O}\right)$. IR: $v$ 1642, 3180, $3213 \mathrm{~cm}^{-1}$. Anal. $\left(\mathrm{C}_{22} \mathrm{H}_{24} \mathrm{~N}_{4} \mathrm{O}_{6} \mathrm{~S}(472.51)\right) \mathrm{C}, \mathrm{H}, \mathrm{N}, \mathrm{S}$. 
2) Preparative and spectroscopic data of derivatives 15, 17, 19, 21 and 23.

5-Chloro-3-[(3,5-dimethylphenyl)sulfonyl]-4-fluoro- $\mathrm{N}$-(pyrrolidin-1-ylmethyl)- $1 \mathrm{H}$-indole-2carboxamide (15). Was synthesized as 13 using 6. Yield 58\%, white solid, mp $119-124{ }^{\circ} \mathrm{C} .{ }^{1} \mathrm{H}$ NMR $\left(\mathrm{DMSO}_{6} \mathrm{~d}_{6}\right): \delta 1.57-1.61(\mathrm{~m}, 4 \mathrm{H}), 2.31(\mathrm{~s}, 6 \mathrm{H}), 2.64-2.68(\mathrm{~m}, 4 \mathrm{H}), 4.29(\mathrm{~d}, J=5.2 \mathrm{~Hz}, 2 \mathrm{H}), 7.24(\mathrm{~s}$, $1 \mathrm{H}), 7.27-7.40(\mathrm{~m}, 2 \mathrm{H}), 7.64(\mathrm{~s}, 2 \mathrm{H}), 9.23\left(\mathrm{br} \mathrm{s}, 1 \mathrm{H}\right.$, disappeared on treatment with $\left.\mathrm{D}_{2} \mathrm{O}\right), 13.01 \mathrm{ppm}$ (br s, $1 \mathrm{H}$, disappeared on treatment with $\left.\mathrm{D}_{2} \mathrm{O}\right)$. IR: $v 1671,3319 \mathrm{~cm}^{-1}$. Anal. $\left(\mathrm{C}_{22} \mathrm{H}_{23} \mathrm{ClFN}_{3} \mathrm{O}_{3} \mathrm{~S}\right.$ (463.95)) C, H, Cl, F, N, S.

5-Bromo-3-[(3,5-dimethylphenyl)sulfonyl]- $N$-(piperidin-1-ylmethyl)-1H-indole-2-

carboxamide (17). Was synthesized as 13 using piperidine. Yield $46 \%$, white solid, $\mathrm{mp} 177-180{ }^{\circ} \mathrm{C}$. ${ }^{1} \mathrm{H}$ NMR (DMSO-d $\left.d_{6}\right): \delta 1.34-1.37(\mathrm{~m}, 2 \mathrm{H}), 1.48-1.52(\mathrm{~m}, 4 \mathrm{H}), 2.31(\mathrm{~s}, 6 \mathrm{H}), 2.54-2.58(\mathrm{~m}, 4 \mathrm{H}), 4.20$ $(\mathrm{d}, J=5.9 \mathrm{~Hz}, 2 \mathrm{H}), 7.27(\mathrm{~s}, 1 \mathrm{H}), 7.43-7.50(\mathrm{~m}, 2 \mathrm{H}), 7.61(\mathrm{~s}, 2 \mathrm{H}), 8.06(\mathrm{~s}, 1 \mathrm{H}), 9.18(\mathrm{br} \mathrm{s}, 1 \mathrm{H}$, disappeared on treatment with $\left.\mathrm{D}_{2} \mathrm{O}\right), 12.95 \mathrm{ppm}\left(\mathrm{br} \mathrm{s}, 1 \mathrm{H}\right.$, disappeared on treatment with $\left.\mathrm{D}_{2} \mathrm{O}\right)$. IR: $v$ $1649,3194,3274 \mathrm{~cm}^{-1}$. Anal. $\left(\mathrm{C}_{23} \mathrm{H}_{26} \mathrm{BrN}_{3} \mathrm{O}_{3} \mathrm{~S}\right.$ (504.44)) C, H, Br, N, S.

5-Chloro-3-[(3,5-dimethylphenyl)sulfonyl]-4-fluoro- $N$-(piperidin-1-ylmethyl)-1H-indole-2carboxamide (19). Was synthesized as 13 using 6 and piperidine. Yield 69\%, white solid, mp 175$177{ }^{\circ} \mathrm{C} .{ }^{1} \mathrm{H}$ NMR (DMSO-d $\left.d_{6}\right): \delta 1.35-1.38(\mathrm{~m}, 2 \mathrm{H}), 1.46-1.51(\mathrm{~m}, 4 \mathrm{H}), 2.32(\mathrm{~s}, 6 \mathrm{H}), 2.63-2.66(\mathrm{~m}$, $4 \mathrm{H}), 4.15(\mathrm{~d}, J=5.9 \mathrm{~Hz}, 2 \mathrm{H}), 7.26(\mathrm{~s}, 1 \mathrm{H}), 7.36-7.43(\mathrm{~m}, 2 \mathrm{H}), 7.63(\mathrm{~s}, 2 \mathrm{H}), 9.24(\mathrm{br} \mathrm{s}, 1 \mathrm{H}$, disappeared on treatment with $\left.\mathrm{D}_{2} \mathrm{O}\right), 13.01 \mathrm{ppm}\left(\mathrm{br} \mathrm{s}, 1 \mathrm{H}\right.$, disappeared on treatment with $\left.\mathrm{D}_{2} \mathrm{O}\right)$. IR: $v$ $1682,3353 \mathrm{~cm}^{-1}$. Anal. $\left(\mathrm{C}_{23} \mathrm{H}_{25} \mathrm{ClFN}_{3} \mathrm{O}_{3} \mathrm{~S}(477.98)\right) \mathrm{C}, \mathrm{H}, \mathrm{Cl}, \mathrm{F}, \mathrm{N}, \mathrm{S}$.

5-Bromo-3-[(3,5-dimethylphenyl)sulfonyl]- $N$-(morpholinomethyl)-1H-indole-2-

carboxamide (21). Was synthesized as 13 using morpholine. Yield 24\%, white solid, mp 267-271 ${ }^{\circ} \mathrm{C}$. ${ }^{1} \mathrm{H}$ NMR (DMSO-d $\left._{6}\right): \delta 2.30(\mathrm{~s}, 6 \mathrm{H}), 2.56-2.58(\mathrm{~m}, 4 \mathrm{H}), 3.57-3.61(\mathrm{~m}, 4 \mathrm{H}), 4.21(\mathrm{~d}, J=5.8 \mathrm{~Hz}$, $2 \mathrm{H}), 7.25(\mathrm{~s}, 1 \mathrm{H}), 7.43-7.49(\mathrm{~m}, 2 \mathrm{H}), 7.63(\mathrm{~s}, 2 \mathrm{H}), 8.05(\mathrm{~s}, 1 \mathrm{H}), 9.24(\mathrm{br} \mathrm{s}, 1 \mathrm{H}$, disappeared on treatment with $\left.\mathrm{D}_{2} \mathrm{O}\right), 13.02 \mathrm{ppm}\left(\mathrm{br} \mathrm{s}, 1 \mathrm{H}\right.$, disappeared on treatment with $\left.\mathrm{D}_{2} \mathrm{O}\right)$. IR: $v 1654,3210 \mathrm{~cm}^{-}$ 1. Anal. $\left(\mathrm{C}_{22} \mathrm{H}_{24} \mathrm{BrN}_{3} \mathrm{O}_{4} \mathrm{~S}(506.41)\right) \mathrm{C}, \mathrm{H}, \mathrm{Br}, \mathrm{N}, \mathrm{S}$.

5-Chloro-3-(3,5-dimethylphenylsulfonyl)-4-fluoro- $\mathrm{N}$-(morpholinomethyl)-1H-indole-2-

carboxamide (23). Was synthesized as 13 using 6 and morpholine. Yield 69\%, white solid, mp 197$199{ }^{\circ} \mathrm{C} .{ }^{1} \mathrm{H}$ NMR (DMSO-d $)$ ): $\delta 2.32(\mathrm{~s}, 6 \mathrm{H}), 2.62-2.64(\mathrm{~m}, 4 \mathrm{H}), 3.58-3.61(\mathrm{~m}, 4 \mathrm{H}), 4.17(\mathrm{~d}, J=6.0$ $\mathrm{Hz}, 2 \mathrm{H}), 7.26(\mathrm{~s}, 1 \mathrm{H}), 7.32-7.38(\mathrm{~m}, 2 \mathrm{H}), 7.66(\mathrm{~s}, 2 \mathrm{H}), 9.31$ (br s, 1H, disappeared on treatment with $\left.\mathrm{D}_{2} \mathrm{O}\right), 13.18 \mathrm{ppm}\left(\right.$ br s, $1 \mathrm{H}$, disappeared on treatment with $\left.\mathrm{D}_{2} \mathrm{O}\right)$. IR: $v 1649,3238 \mathrm{~cm}^{-1}$. Anal. $\left(\mathrm{C}_{22} \mathrm{H}_{23} \mathrm{ClFN}_{3} \mathrm{O}_{4} \mathrm{~S}(479.95)\right) \mathrm{C}, \mathrm{H}, \mathrm{Cl}, \mathrm{F}, \mathrm{N}, \mathrm{S}$. 
3) Preparative and spectroscopic data of derivatives $25,26,29,30,32-34,36-38,40-42$ and 44-46.

$\mathrm{N}$-Benzyl-5-bromo-3-[(3,5-dimethylphenyl)sulfonyl]-1H-indole-2-carboxamide (25). Was synthesized as 24 using 51 and benzylamine. Yield 50\%, white solid, mp 266-269 ${ }^{\circ} \mathrm{C}$ (from ethanol). ${ }^{1} \mathrm{H}$ NMR (DMSO- $\left.d_{6}\right): \delta 2.26(\mathrm{~s}, 6 \mathrm{H}), 4.58(\mathrm{~d}, J=5.6 \mathrm{~Hz}, 2 \mathrm{H}), 7.24(\mathrm{~s}, 1 \mathrm{H}), 7.27(\mathrm{t}, J=7.7 \mathrm{~Hz}, 1 \mathrm{H})$, $7.34(\mathrm{t}, J=7.1 \mathrm{~Hz}, 2 \mathrm{H}), 7.43-7.50(\mathrm{~m}, 4 \mathrm{H}), 7.58(\mathrm{~s}, 2 \mathrm{H}), 8.08(\mathrm{~s}, 1 \mathrm{H}), 9.42(\mathrm{br} \mathrm{s}, 1 \mathrm{H}$, disappeared on treatment with $\left.\mathrm{D}_{2} \mathrm{O}\right), 13.03 \mathrm{ppm}\left(\mathrm{br} \mathrm{s}, 1 \mathrm{H}\right.$, disappeared on treatment with $\left.\mathrm{D}_{2} \mathrm{O}\right)$. IR: $v 1645,3214 \mathrm{~cm}^{-}$ 1. Anal. $\left(\mathrm{C}_{24} \mathrm{H}_{21} \mathrm{BrN}_{2} \mathrm{O}_{3} \mathrm{~S}(497.40)\right) \mathrm{C}, \mathrm{H}, \mathrm{Br}, \mathrm{N}, \mathrm{S}$.

$N$-Benzyl-3-[(3,5-dimethylphenyl)sulfonyl]-5-nitro-1H-indole-2-carboxamide (26). Was synthesized as 24 from 52 using benzylamine. Yield 55\%, white solid, mp $246-248{ }^{\circ} \mathrm{C}$ (from ethanol). ${ }^{1} \mathrm{H}$ NMR (DMSO-d $\left.{ }_{6}\right): \delta 2.26(\mathrm{~s}, 6 \mathrm{H}), 4.58(\mathrm{~d}, J=5.7 \mathrm{~Hz}, 2 \mathrm{H}), 7.25(\mathrm{~s}, 1 \mathrm{H}), 7.29(\mathrm{t}, J=7.2$ $\mathrm{Hz}, 1 \mathrm{H}), 7.37(\mathrm{t}, J=7.5 \mathrm{~Hz}, 2 \mathrm{H}), 7.45(\mathrm{~d}, J=7.3 \mathrm{~Hz}, 2 \mathrm{H}), 7.61(\mathrm{~s}, 2 \mathrm{H}), 7.70(\mathrm{~d}, J=9.1 \mathrm{~Hz}, 1 \mathrm{H}), 8.17$ (dd, $J=2.3$ and $9.0 \mathrm{~Hz}, 1 \mathrm{H}), 8.83(\mathrm{~d}, J=2.3 \mathrm{~Hz}, 1 \mathrm{H}) 9.47$ (br s, $1 \mathrm{H}$, disappeared on treatment with $\mathrm{D}_{2} \mathrm{O}$ ), 13.50 ppm (br s, $1 \mathrm{H}$, disappeared on treatment with $\mathrm{D}_{2} \mathrm{O}$ ). IR: $v 1637,3190,3281 \mathrm{~cm}^{-1}$. Anal. $\left(\mathrm{C}_{24} \mathrm{H}_{21} \mathrm{~N}_{3} \mathrm{O}_{5} \mathrm{~S}(463.51)\right) \mathrm{C}, \mathrm{H}, \mathrm{N}, \mathrm{S}$.

5-Bromo-3-[(3,5-dimethylphenyl)sulfonyl]- $N$-(2-(pyrrolidin-1-yl)ethyl)-1H-indole-2carboxamide (29). Was synthesized as 24 using 51 and 2-(pyrrolidin-1-yl)ethanamine. Yield 70\%, white solid, mp 207-210 ${ }^{\circ} \mathrm{C}$ (from ethanol). ${ }^{1} \mathrm{H}$ NMR (DMSO- $\left.d_{6}\right): \delta 1.67-1.71(\mathrm{~m}, 4 \mathrm{H}), 2.30(\mathrm{~s}, 6 \mathrm{H})$, 2.52-2.56 (m, 4H), $2.66(\mathrm{t}, J=6.8 \mathrm{~Hz}, 2 \mathrm{H}), 3.47(\mathrm{q}, J=6.9 \mathrm{~Hz}, 2 \mathrm{H}), 7.25(\mathrm{~s}, 1 \mathrm{H}), 7.38-7.48(\mathrm{~m}, 2 \mathrm{H})$, $7.61(\mathrm{~s}, 2 \mathrm{H}), 8.1(\mathrm{~s}, 1 \mathrm{H}), 9.00\left(\mathrm{br} \mathrm{s}, 1 \mathrm{H}\right.$, disappeared on treatment with $\left.\mathrm{D}_{2} \mathrm{O}\right), 13.05 \mathrm{ppm}(\mathrm{br} \mathrm{s}, 1 \mathrm{H}$, disappeared on treatment with $\left.\mathrm{D}_{2} \mathrm{O}\right)$. IR: $v 1648,3204 \mathrm{~cm}^{-1}$. Anal. $\left(\mathrm{C}_{23} \mathrm{H}_{26} \mathrm{BrN}_{3} \mathrm{O}_{3} \mathrm{~S}(504.44)\right) \mathrm{C}, \mathrm{H}$, $\mathrm{Br}, \mathrm{N}, \mathrm{S}$.

3-[(3,5-Dimethylphenyl)sulfonyl]-5-nitro- $N$-(2-(pyrrolidin-1-yl)ethyl)-1H-indole-2carboxamide (30). Was synthesized as 24 using 52 and 2-(pyrrolidin-1-yl)ethanamine. Yield 46\%, white solid, mp $216-218^{\circ} \mathrm{C}$ (from ethanol). ${ }^{1} \mathrm{H}$ NMR (DMSO- $\left.d_{6}\right): \delta 1.74-1.78(\mathrm{~m}, 4 \mathrm{H}), 2.28(\mathrm{~s}, 6 \mathrm{H})$, 2.81-2.85 (m, 4H), $2.90(\mathrm{t}, J=6.3 \mathrm{~Hz}, 2 \mathrm{H}), 3.50(\mathrm{q}, J=6.2 \mathrm{~Hz}, 2 \mathrm{H}), 7.20(\mathrm{~s}, 1 \mathrm{H}), 7.57-7.62(\mathrm{~m}, 3 \mathrm{H})$, $8.02(\mathrm{dd}, J=2.1$ and $9.0 \mathrm{~Hz}, 1 \mathrm{H}), 8.84-8.89(\mathrm{~m}, 2 \mathrm{H}), 13.46 \mathrm{ppm}(\mathrm{br} \mathrm{s}, 1 \mathrm{H}$, disappeared on treatment with $\left.\mathrm{D}_{2} \mathrm{O}\right)$. IR: $v 1643,3181 \mathrm{~cm}^{-1}$. Anal. $\left(\mathrm{C}_{23} \mathrm{H}_{26} \mathrm{~N}_{4} \mathrm{O}_{5} \mathrm{~S}(470.54)\right) \mathrm{C}, \mathrm{H}, \mathrm{N}, \mathrm{S}$.

5-Chloro-3-[(3,5-dimethylphenyl)sulfonyl]- $N$-(2-morpholinoethyl)-1H-indole-2-

carboxamide (32). Was synthesized as 24 using 2-morpholin-4-ylethanamine. Yield 25\%, white solid, mp 208-210 ${ }^{\circ} \mathrm{C}$ (from ethanol). ${ }^{1} \mathrm{H}$ NMR (DMSO-d 6 ): $\delta 2.31(\mathrm{~s}, 6 \mathrm{H}), 2.48-2.53(\mathrm{~m}, 4 \mathrm{H}), 3.50-$ $3.59(\mathrm{~m}, 8 \mathrm{H}), 7.28(\mathrm{~s}, 1 \mathrm{H}), 7.34(\mathrm{~d}, J=9.0 \mathrm{~Hz}, 1 \mathrm{H}), 7.54(\mathrm{~d}, J=8.12 \mathrm{~Hz}, 1 \mathrm{H}), 7.63(\mathrm{~s}, 2 \mathrm{H}), 7.97(\mathrm{~s}$, 1H), 9.09 (br s, 1H, disappeared on treatment with $\mathrm{D}_{2} \mathrm{O}$ ), $13.04 \mathrm{ppm}$ (br s, 1H, disappeared on treatment with $\left.\mathrm{D}_{2} \mathrm{O}\right)$. IR: $v$ 1646, $3213 \mathrm{~cm}^{-1}$. Anal. $\left(\mathrm{C}_{23} \mathrm{H}_{26} \mathrm{ClN}_{3} \mathrm{O}_{4} \mathrm{~S}(475.99)\right) \mathrm{C}, \mathrm{H}, \mathrm{Cl}, \mathrm{N}, \mathrm{S}$.

5-Bromo-3-[(3,5-dimethylphenyl)sulfonyl]- $N$-(2-morpholinoethyl)-1H-indole-2-

carboxamide (33). Was synthesized as $\mathbf{2 4}$ using $\mathbf{5 1}$ and 2-morpholin-4-yl-ethanamine. Yield 53\%, white solid, mp 218-223 ${ }^{\circ} \mathrm{C}$ (from ethanol). ${ }^{1} \mathrm{H}$ NMR (DMSO- $\left.d_{6}\right): \delta 2.30(\mathrm{~s}, 6 \mathrm{H}), 2.43-2.47(\mathrm{~m}, 4 \mathrm{H})$, $2.53(\mathrm{t}, J=6.4 \mathrm{~Hz}, 2 \mathrm{H}), 3.49(\mathrm{q}, J=6.0 \mathrm{~Hz}, 2 \mathrm{H}), 3.56-3.59(\mathrm{~m}, 4 \mathrm{H}), 7.26(\mathrm{~s}, 1 \mathrm{H}), 7.43-7.49(\mathrm{~m}, 2 \mathrm{H})$, $7.60(\mathrm{~s}, 2 \mathrm{H}), 8.10(\mathrm{~s}, 1 \mathrm{H}), 9.06\left(\mathrm{br} \mathrm{s}, 1 \mathrm{H}\right.$, disappeared on treatment with $\left.\mathrm{D}_{2} \mathrm{O}\right), 13.01 \mathrm{ppm}(\mathrm{br} \mathrm{s}, 1 \mathrm{H}$, disappeared on treatment with $\left.\mathrm{D}_{2} \mathrm{O}\right)$. IR: $v 1640,3196 \mathrm{~cm}^{-1}$. Anal. $\left(\mathrm{C}_{23} \mathrm{H}_{26} \mathrm{BrN}_{3} \mathrm{O}_{4} \mathrm{~S}(520.44)\right) \mathrm{C}, \mathrm{H}$, $\mathrm{Br}, \mathrm{N}, \mathrm{S}$.

3-(3,5-Dimethylphenylsulfonyl)- $N$-(2-morpholinoethyl)-5-nitro-1 $H$-indole-2-carboxamide (34). Was synthesized as 24 using 52 and 2-morpholin-4-yl-ethanamine. Yield 63\%, yellow solid, mp 243-246 ${ }^{\circ} \mathrm{C}$ (from ethanol). ${ }^{1} \mathrm{H}$ NMR (DMSO- $\left.d_{6}\right): \delta 2.30(\mathrm{~s}, 6 \mathrm{H}), 2.46-2.49(\mathrm{~m}, 4 \mathrm{H}), 2.55(\mathrm{t}, J=6.4$ $\mathrm{Hz}, 2 \mathrm{H}), 3.50(\mathrm{q}, J=6.0 \mathrm{~Hz}, 2 \mathrm{H}), 3.58-3.60(\mathrm{~m}, 4 \mathrm{H}), 7.27(\mathrm{~s}, 1 \mathrm{H}), 7.63(\mathrm{~s}, 2 \mathrm{H}), 7.69(\mathrm{~d}, J=9.1 \mathrm{~Hz}$, $1 \mathrm{H}), 8.17(\mathrm{dd}, J=1.8$ and $8.9 \mathrm{~Hz}, 1 \mathrm{H}), 8.86(\mathrm{~d}, J=2.1 \mathrm{~Hz}, 1 \mathrm{H}), 9.05$ (br s, $1 \mathrm{H}$, disappeared on treatment with $\mathrm{D}_{2} \mathrm{O}$ ), $13.46 \mathrm{ppm}$ (br s, $1 \mathrm{H}$, disappeared on treatment with $\mathrm{D}_{2} \mathrm{O}$ ). IR: $v 1648,3163 \mathrm{~cm}^{-}$ 1. Anal. $\left(\mathrm{C}_{23} \mathrm{H}_{26} \mathrm{~N}_{4} \mathrm{O}_{6} \mathrm{~S}(486.54)\right) \mathrm{C}, \mathrm{H}, \mathrm{N}, \mathrm{S}$. 
5-Chloro-3-[(3,5-dimethylphenyl)sulfonyl]- $N$-phenethyl-1H-indole-2-carboxamide (36). Was synthesized as $\mathbf{2 4}$ using 2-phenylethanamine. Yield $60 \%$, white solid, mp $227-228{ }^{\circ} \mathrm{C}$ (from ethanol). ${ }^{1} \mathrm{H}$ NMR (DMSO- $\left.d_{6}\right): \delta 2.32(\mathrm{~s}, 6 \mathrm{H}), 2.91(\mathrm{t}, J=7.4 \mathrm{~Hz}, 2 \mathrm{H}), 3.58-3.63(\mathrm{~m}, 2 \mathrm{H}), 7.23-7.37(\mathrm{~m}, 7 \mathrm{H})$, $7.47(\mathrm{~d}, J=7.1 \mathrm{~Hz}, 1 \mathrm{H}), 7.52(\mathrm{~s}, 2 \mathrm{H}), 7.94(\mathrm{~d}, J=2.0 \mathrm{~Hz}, 1 \mathrm{H}), 9.10$ (br s, 1H, disappeared on treatment with $\left.\mathrm{D}_{2} \mathrm{O}\right), 13.05 \mathrm{ppm}$ (br s, $1 \mathrm{H}$, disappeared on treatment with $\mathrm{D}_{2} \mathrm{O}$ ). IR: $v 1643,3195$, $3287 \mathrm{~cm}^{-1}$. Anal. $\left(\mathrm{C}_{25} \mathrm{H}_{23} \mathrm{ClN}_{2} \mathrm{O}_{3} \mathrm{~S}(466.98)\right) \mathrm{C}, \mathrm{H}, \mathrm{Cl}, \mathrm{N}, \mathrm{S}$.

5-Bromo-3-[(3,5-dimethylphenyl)sulfonyl]- $N$-phenethyl-1H-indole-2-carboxamide (37). Was synthesized as $\mathbf{2 4}$ using 51 and 2-phenylethanamine. Yield $30 \%$, white solid, mp $231-236{ }^{\circ} \mathrm{C}$ (from ethanol). ${ }^{1} \mathrm{H}$ NMR (DMSO-d $\left.d_{6}\right): \delta 2.29(\mathrm{~s}, 6 \mathrm{H}), 2.90(\mathrm{t}, J=7.4 \mathrm{~Hz}, 2 \mathrm{H}), 3.58(\mathrm{q}, J=6.7 \mathrm{~Hz}, 2 \mathrm{H})$, 7.22-7.31 (m, 6H), 7.43-7.49 (m, 2H), $7.59(\mathrm{~s}, 2 \mathrm{H}), 8.07(\mathrm{~s}, 1 \mathrm{H}), 9.08$ (br s, 1H, disappeared on treatment with $\left.\mathrm{D}_{2} \mathrm{O}\right), 13.00 \mathrm{ppm}\left(\mathrm{br} \mathrm{s}, 1 \mathrm{H}\right.$, disappeared on treatment with $\left.\mathrm{D}_{2} \mathrm{O}\right)$. IR: $v$ 1640, 3191, $3290 \mathrm{~cm}^{-1}$. Anal. $\left(\mathrm{C}_{25} \mathrm{H}_{23} \mathrm{BrN}_{2} \mathrm{O}_{3} \mathrm{~S}(511.43)\right) \mathrm{C}, \mathrm{H}, \mathrm{Br}, \mathrm{N}, \mathrm{S}$.

3-[(3,5-Dimethylphenyl)sulfonyl]-5-nitro- $N$-phenethyl-1H-indole-2-carboxamide (38). Was synthesized as 24 using 52 and 2-phenylethanamine. Yield $74 \%$, white solid, mp $261-262{ }^{\circ} \mathrm{C}$ (from ethanol). ${ }^{1} \mathrm{H}$ NMR (DMSO-d $\left.d_{6}\right): \delta 2.29(\mathrm{~s}, 6 \mathrm{H}), 2.89(\mathrm{t}, J=7.4 \mathrm{~Hz}, 2 \mathrm{H}), 3.56(\mathrm{q}, J=6.7 \mathrm{~Hz}, 2 \mathrm{H})$, 7.20-7.24 (m, 3H), 7.28-7.31 (m, 4H), 7.63-7.67 (m, 2H), $8.11(\mathrm{dd}, J=1.9$ and $8.9 \mathrm{~Hz}, 1 \mathrm{H}), 8.85(\mathrm{~d}, J$ $=1.9 \mathrm{~Hz}, 1 \mathrm{H}), 8.98\left(\mathrm{br} \mathrm{s}, 1 \mathrm{H}\right.$, disappeared on treatment with $\left.\mathrm{D}_{2} \mathrm{O}\right), 13.47 \mathrm{ppm}(\mathrm{br} \mathrm{s}, 1 \mathrm{H}$, disappeared on treatment with $\left.\mathrm{D}_{2} \mathrm{O}\right)$. IR: $v$ 1641, 3176, $3284 \mathrm{~cm}^{-1}$. Anal. $\left(\mathrm{C}_{25} \mathrm{H}_{23} \mathrm{~N}_{3} \mathrm{O}_{5} \mathrm{~S}(477.53)\right) \mathrm{C}, \mathrm{H}, \mathrm{N}, \mathrm{S}$.

$\mathrm{N}$-[2-(1H-Pyrrol-1-yl)ethyl]-5-chloro-3-[(3,5-dimethylphenyl)sulfonyl]-1H-indole-2-

carboxamide (40). Was synthesized as 24 using 2-(1H-pyrrol-1-yl)ethylamine. Yield $38 \%$, white solid, mp 200-203 ${ }^{\circ} \mathrm{C}$ (from ethanol). ${ }^{1} \mathrm{H}$ NMR (DMSO-d $)$ ): $\delta 2.31(\mathrm{~s}, 6 \mathrm{H}), 3.66(\mathrm{q}, J=6.1 \mathrm{~Hz}, 2 \mathrm{H})$, $4.14(\mathrm{t}, J=6.2 \mathrm{~Hz}, 2 \mathrm{H}), 6.01(\mathrm{t}, J=2.1 \mathrm{~Hz}, 2 \mathrm{H}), 6.87(\mathrm{t}, J=2.1 \mathrm{~Hz}, 2 \mathrm{H}), 7.26(\mathrm{~s}, 1 \mathrm{H}), 7.34(\mathrm{dd}, J=$ 2.0 and $8.8 \mathrm{~Hz}, 1 \mathrm{H}), 7.54(\mathrm{~d}, J=8.8 \mathrm{~Hz}, 1 \mathrm{H}), 7.65(\mathrm{~s}, 2 \mathrm{H}), 7.91(\mathrm{~d}, J=1.9 \mathrm{~Hz}, 1 \mathrm{H}), 9.18$ (br s, $1 \mathrm{H}$, disappeared on treatment with $\left.\mathrm{D}_{2} \mathrm{O}\right), 13.08 \mathrm{ppm}\left(\mathrm{br} \mathrm{s}, 1 \mathrm{H}\right.$, disappeared on treatment with $\left.\mathrm{D}_{2} \mathrm{O}\right)$. IR: $v$ $1638,3215 \mathrm{~cm}^{-1}$. Anal. $\left(\mathrm{C}_{23} \mathrm{H}_{22} \mathrm{ClN}_{3} \mathrm{O}_{3} \mathrm{~S}(455.96)\right) \mathrm{C}, \mathrm{H}, \mathrm{Cl}, \mathrm{N}, \mathrm{S}$.

$\mathrm{N}$-[2-(1H-Pyrrol-1-yl)ethyl]-5-bromo-3-[(3,5-dimethylphenyl)sulfonyl]-1H-indole-2-

carboxamide (41). Was synthesized as 24 using 51 and 2-(1H-pyrrol-1-yl)ethylamine. Yield 29\%, white solid, mp 213-216 ${ }^{\circ} \mathrm{C}$ (from ethanol). ${ }^{1} \mathrm{H}$ NMR (DMSO- $\left.d_{6}\right): \delta 2.30(\mathrm{~s}, 6 \mathrm{H}), 3.65(\mathrm{q}, J=5.2 \mathrm{~Hz}$, $2 \mathrm{H}), 4.12(\mathrm{t}, J=6.4 \mathrm{~Hz}, 2 \mathrm{H}), 5.99(\mathrm{~s}, 2 \mathrm{H}), 6.84(\mathrm{~s}, 2 \mathrm{H}), 7.25(\mathrm{~s}, 1 \mathrm{H}), 7.43-7.49(\mathrm{~m}, 2 \mathrm{H}), 7.16(\mathrm{~s}, 2 \mathrm{H})$, $8.04(\mathrm{~s}, 1 \mathrm{H}), 9.14$ (br s, $1 \mathrm{H}$, disappeared on treatment with $\left.\mathrm{D}_{2} \mathrm{O}\right), 13.00 \mathrm{ppm}(\mathrm{br} \mathrm{s}, 1 \mathrm{H}$, disappeared on treatment with $\left.\mathrm{D}_{2} \mathrm{O}\right)$. IR: $v$ 1655, $3195 \mathrm{~cm}^{-1}$. Anal. $\left(\mathrm{C}_{23} \mathrm{H}_{22} \mathrm{BrN}_{3} \mathrm{O}_{3} \mathrm{~S}(500.41)\right) \mathrm{C}, \mathrm{H}, \mathrm{Br}, \mathrm{N}, \mathrm{S}$.

$\mathrm{N}$-[2-(1H-Pyrrol-1-yl)ethyl]-3-[(3,5-dimethylphenyl)sulfonyl]-5-nitro-1H-indole-2-

carboxamide (42). Was synthesized as 24 using 52 and 2-(1H-pyrrol-1-yl)ethylamine. Yield 76\%, yellow solid, mp $234-237{ }^{\circ} \mathrm{C}$ (from ethanol). ${ }^{1} \mathrm{H}$ NMR (DMSO- $d_{6}$ ): $\delta 2.30(\mathrm{~s}, 6 \mathrm{H}), 3.65$ (q, $J=5.9$ $\mathrm{Hz}, 2 \mathrm{H}), 4.12(\mathrm{t}, J=6.1 \mathrm{~Hz}, 2 \mathrm{H}), 5.99(\mathrm{~s}, 2 \mathrm{H}), 6.85(\mathrm{~s}, 2 \mathrm{H}), 7.27(\mathrm{~s}, 1 \mathrm{H}), 7.65(\mathrm{~s}, 2 \mathrm{H}), 7.69(\mathrm{~d}, J=9.1$ $\mathrm{Hz}, 1 \mathrm{H}), 8.16(\mathrm{dd}, J=1.7$ and $9.2 \mathrm{~Hz}, 1 \mathrm{H}), 8.80(\mathrm{~d}, J=1.9 \mathrm{~Hz}, 1 \mathrm{H}), 9.18$ (br s, $1 \mathrm{H}$, disappeared on treatment with $\left.\mathrm{D}_{2} \mathrm{O}\right), 13.44 \mathrm{ppm}\left(\mathrm{br} \mathrm{s}, 1 \mathrm{H}\right.$, disappeared on treatment with $\left.\mathrm{D}_{2} \mathrm{O}\right)$. IR: $v 1648,3228 \mathrm{~cm}^{-}$ 1. Anal. $\left(\mathrm{C}_{23} \mathrm{H}_{22} \mathrm{~N}_{4} \mathrm{O}_{5} \mathrm{~S}(466.51)\right) \mathrm{C}, \mathrm{H}, \mathrm{N}, \mathrm{S}$.

5-Chloro-3-[(3,5-dimethylphenyl)sulfonyl]- $N$-(2-phenoxyethyl)-1H-indole-2-carboxamide (44). Was synthesized as 24 using 2-phenoxyethanamine. Yield $83 \%$, white solid, mp 227-228 ${ }^{\circ} \mathrm{C}$ (from ethanol). ${ }^{1} \mathrm{H}$ NMR $\left(\mathrm{CDCl}_{3}\right): \delta 2.17(2,6 \mathrm{H}), 3.97(\mathrm{q}, J=6.3 \mathrm{~Hz}, 2 \mathrm{H}), 4.23(\mathrm{t}, J=5.2 \mathrm{~Hz}, 2 \mathrm{H})$, 6.95-7.00 (m, 3H), $7.09(\mathrm{~s}, 1 \mathrm{H}), 7.26-7.34(\mathrm{~m}, 3 \mathrm{H}), 7.39(\mathrm{~d}, J=8.8 \mathrm{~Hz}, 1 \mathrm{H}), 7.51(\mathrm{~s}, 2 \mathrm{H}), 8.28(\mathrm{~d}, J=$ $1.8 \mathrm{~Hz}, 1 \mathrm{H}), 10.05$ (br s, $1 \mathrm{H}$, disappeared with $\left.\mathrm{D}_{2} \mathrm{O}\right), 10.31 \mathrm{ppm}\left(\right.$ br s, $1 \mathrm{H}$, disappeared with $\left.\mathrm{D}_{2} \mathrm{O}\right)$. IR: v 1650, $3209 \mathrm{~cm}^{-1}$. Anal. $\left(\mathrm{C}_{25} \mathrm{H}_{23} \mathrm{ClN}_{2} \mathrm{O}_{4} \mathrm{~S}(482.98)\right) \mathrm{C}, \mathrm{H}, \mathrm{Cl}, \mathrm{N}, \mathrm{S}$.

5-Bromo-3-[(3,5-dimethylphenyl)sulfonyl]- $N$-(2-phenoxyethyl)-1 $\mathrm{H}$-indole-2-carboxamide (45). Was synthesized as 24 using 51 and 2-phenoxyethanamine. Yield 52\%, white solid, mp 214-219 ${ }^{\circ} \mathrm{C}$ (from ethanol). ${ }^{1} \mathrm{H}$ NMR (DMSO- $\left.d_{6}\right): \delta 2.18(\mathrm{~s}, 6 \mathrm{H}), 4.62(\mathrm{q}, J=6.2 \mathrm{~Hz}, 2 \mathrm{H}), 4.07$ (t, $J=6.4 \mathrm{~Hz}$, 
2H), 6.88-6.97 (m, 3H), 7.04-7.08 (m, 2H), 7.26-7.37 (m, 3H), $7.55(\mathrm{~s}, 2 \mathrm{H}), 8.09(\mathrm{~s}, 1 \mathrm{H}), 8.50$ (br s, $1 \mathrm{H}$, disappeared on treatment with $\left.\mathrm{D}_{2} \mathrm{O}\right), 13.05 \mathrm{ppm}\left(\mathrm{br} \mathrm{s}, 1 \mathrm{H}\right.$, disappeared on treatment with $\mathrm{D}_{2} \mathrm{O}$ ). IR: v 1644, $3206 \mathrm{~cm}^{-1}$. Anal. $\left(\mathrm{C}_{25} \mathrm{H}_{23} \mathrm{BrN}_{2} \mathrm{O}_{4} \mathrm{~S}(527.43)\right) \mathrm{C}, \mathrm{H}, \mathrm{Br}, \mathrm{N}, \mathrm{S}$.

3-[(3,5-Dimethylphenyl)sulfonyl]-5-nitro- $N$-(2-phenoxyethyl)- $1 \mathrm{H}$-indole-2-carboxamide (46). Was synthesized as 24 using 52 and 2-phenoxyethanamine. Yield 93\%, white solid, mp 278-280 ${ }^{\circ} \mathrm{C}$ (from ethanol). ${ }^{1} \mathrm{H}$ NMR (DMSO- $d_{6}$ ): $\delta 2.23(\mathrm{~s}, 6 \mathrm{H}), 3.77$ (q, $\left.J=5.4 \mathrm{~Hz}, 2 \mathrm{H}\right), 4.18(\mathrm{t}, J=5.2 \mathrm{~Hz}$, 2H), 6.93-7.00 (m, 3H), $7.24(\mathrm{~s}, 1 \mathrm{H}), 7.30(\mathrm{t}, J=7.3 \mathrm{~Hz}, 2 \mathrm{H}), 7.62(\mathrm{~s}, 2 \mathrm{H}), 7.70(\mathrm{~d}, J=9.1 \mathrm{~Hz}, 1 \mathrm{H})$, $8.18(\mathrm{dd}, J=1.8$ and $8.8 \mathrm{~Hz}, 1 \mathrm{H}), 8.88(\mathrm{~d}, J=2.2 \mathrm{~Hz}, 1 \mathrm{H}), 9.33$ (br s, $1 \mathrm{H}$, disappeared on treatment with $\left.\mathrm{D}_{2} \mathrm{O}\right), 13.48 \mathrm{ppm}\left(\right.$ br s, $1 \mathrm{H}$, disappeared on treatment with $\left.\mathrm{D}_{2} \mathrm{O}\right)$. IR: $v 1642,3188,3279 \mathrm{~cm}^{-1}$. Anal. $\left(\mathrm{C}_{25} \mathrm{H}_{23} \mathrm{~N}_{3} \mathrm{O}_{6} \mathrm{~S}(493.53)\right) \mathrm{C}, \mathrm{H}, \mathrm{N}, \mathrm{S}$. 
4) Preparative and spectroscopic data of derivatives $28,31,35,39,43$ and 47.

5-Chloro-3-[(3,5-dimethylphenyl)sulfonyl]- $N$-[2-(pyrrolidin-1-yl)ethyl]-1H-indole-2-

carboxamide (28). Was synthesized as 27 using 54 and 2-(pyrrolidin-1-yl)ethanamine. Yield 82\%, white solid, mp 200-203 ${ }^{\circ} \mathrm{C}$ (from ethanol). ${ }^{1} \mathrm{H}$ NMR (DMSO- $\left.d_{6}\right): \delta 2.29(\mathrm{~s}, 6 \mathrm{H}), 2.54(\mathrm{~m}, 8 \mathrm{H}), 2.66$ (t, $J=6.6 \mathrm{~Hz}, 2 \mathrm{H}), 3.40(\mathrm{q}, J=6.1 \mathrm{~Hz}, 2 \mathrm{H}), 7.25(\mathrm{~s}, 1 \mathrm{H}), 7.31(\mathrm{dd}, J=1.9$ and $8.8 \mathrm{~Hz}, 1 \mathrm{H}), 7.51(\mathrm{~d}, J$ $=8.7 \mathrm{~Hz}, 1 \mathrm{H}), 7.61(\mathrm{~s}, 2 \mathrm{H}), 7.94(\mathrm{~d}, J=1.7 \mathrm{~Hz}, 1 \mathrm{H}), 9.00(\mathrm{br} \mathrm{s}, 1 \mathrm{H}$, disappeared on treatment with $\mathrm{D}_{2} \mathrm{O}$ ), $13.02 \mathrm{ppm}\left(\right.$ br s, $1 \mathrm{H}$, disappeared on treatment with $\left.\mathrm{D}_{2} \mathrm{O}\right)$. IR: $v 1648,3204 \mathrm{~cm}^{-1}$. Anal. $\left(\mathrm{C}_{23} \mathrm{H}_{26} \mathrm{ClN}_{3} \mathrm{O}_{3} \mathrm{~S}(459.99)\right) \mathrm{C}, \mathrm{H}, \mathrm{Cl}, \mathrm{N}, \mathrm{S}$.

5-Chloro-3-[(3,5-dimethylphenyl)sulfonyl]-4-fluoro- $N$-[2-(pyrrolidin-1-yl)ethyl]-1H-indole2-carboxamide (31). Was synthesized as 27 using 2-(pyrrolidin-1-yl)ethanamine. Yield 59\%, white solid, mp 194-195 ${ }^{\circ} \mathrm{C}$ (from ethanol). ${ }^{1} \mathrm{H}$ NMR (DMSO- $\left.d_{6}\right): \delta 1.69-1.71(\mathrm{~m}, 4 \mathrm{H}), 2.32(\mathrm{~s}, 6 \mathrm{H}), 2.55-$ $2.58(\mathrm{~m}, 4 \mathrm{H}), 2.68(\mathrm{t}, J=7.0 \mathrm{~Hz}, 2 \mathrm{H}), 3.43(\mathrm{q}, J=6.5 \mathrm{~Hz}, 2 \mathrm{H}), 7.24(\mathrm{~s}, 1 \mathrm{H}), 7.29-7.32(\mathrm{~m}, 2 \mathrm{H}), 7.67$ $(\mathrm{s}, 2 \mathrm{H}), 8.92$ (br s, $1 \mathrm{H}$, disappeared on treatment with $\left.\mathrm{D}_{2} \mathrm{O}\right), 13.21 \mathrm{ppm}($ br s, $1 \mathrm{H}$, disappeared on treatment with $\left.\mathrm{D}_{2} \mathrm{O}\right)$. IR: $v$ 1655, $3344 \mathrm{~cm}^{-1}$. Anal. $\left(\mathrm{C}_{23} \mathrm{H}_{25} \mathrm{ClFN}_{3} \mathrm{O}_{3} \mathrm{~S}(477.98)\right) \mathrm{C}, \mathrm{H}, \mathrm{Cl}, \mathrm{F}, \mathrm{N}, \mathrm{S}$.

5-Chloro-3-[(3,5-dimethylphenyl)sulfonyl]-4-fluoro- $N$-[2-(morpholin-4-yl) ethyl]-1 $\mathrm{H}$-indole2-carboxamide (35). Was synthesized as 27 using 2-(morpholin-4-yl)ethanamine. Yield 93\%, white solid, mp 216-217 ${ }^{\circ} \mathrm{C}$ (from ethanol). ${ }^{1} \mathrm{H}$ NMR $\left(\mathrm{CDCl}_{3}\right): \delta 2.37(\mathrm{~s}, 6 \mathrm{H}), 2.54-2.56(\mathrm{~m}, 4 \mathrm{H}), 2.68(\mathrm{t}, J$ $=6.1 \mathrm{~Hz}, 2 \mathrm{H}), 3.69-3.71(\mathrm{~m}, 6 \mathrm{H}), 7.20(\mathrm{~s}, 1 \mathrm{H}), 7.22(\mathrm{~s}, 1 \mathrm{H}), 7.33(\mathrm{dd}, J=6.4$ and $8.8 \mathrm{~Hz}, 1 \mathrm{H}), 7.59$ $(\mathrm{s}, 2 \mathrm{H}), 10.14$ (br s, 1H, disappeared on treatment with $\left.\mathrm{D}_{2} \mathrm{O}\right), 10.38 \mathrm{ppm}$ (br s, 1H, disappeared on treatment with $\left.\mathrm{D}_{2} \mathrm{O}\right)$. IR: $v$ 1646, 3181, $3266 \mathrm{~cm}^{-1}$. Anal. $\left(\mathrm{C}_{23} \mathrm{H}_{25} \mathrm{ClFN}_{3} \mathrm{O}_{4} \mathrm{~S}(493.98)\right) \mathrm{C}, \mathrm{H}, \mathrm{Cl}, \mathrm{F}, \mathrm{N}$, S.

5-Chloro-3-[(3,5-dimethylphenyl)sulfonyl]-4-fluoro- $N$-phenethyl-1H-indole-2-carboxamide (39). Was synthesized as 27 using 2-phenylethanamine. Yield $88 \%$, white solid, mp 184-185 ${ }^{\circ} \mathrm{C}$ (from ethanol). ${ }^{1} \mathrm{H}$ NMR (DMSO- $\left.d_{6}\right): \delta 2.32(\mathrm{~s}, 6 \mathrm{H}), 2.90(\mathrm{t}, J=7.5 \mathrm{~Hz}, 2 \mathrm{H}), 3.54(\mathrm{q}, J=5.5 \mathrm{~Hz}$, $2 \mathrm{H}), 7.22-7.42(\mathrm{~m}, 8 \mathrm{H}), 7.66(\mathrm{~s}, 2 \mathrm{H}), 9.16\left(\mathrm{br} \mathrm{s}, 1 \mathrm{H}\right.$, disappeared on treatment with $\left.\mathrm{D}_{2} \mathrm{O}\right), 13.24 \mathrm{ppm}$ (br s, $1 \mathrm{H}$, disappeared on treatment with $\left.\mathrm{D}_{2} \mathrm{O}\right)$. IR: $v 1649,3197 \mathrm{~cm}^{-1}$. Anal. $\left(\mathrm{C}_{25} \mathrm{H}_{22} \mathrm{ClFN}_{2} \mathrm{O}_{3} \mathrm{~S}\right.$ (484.97)) C, H, Cl, F, N, S.

$\mathrm{N}$-[2-(1H-Pyrrol-1-yl)ethyl]-5-chloro-3-[(3,5-dimethylphenyl)sulfonyl]-4-fluoro-1H-indole-2carboxamide (43). Was synthesized as 27 using 2-(1H-pyrrol-1-yl)ethanamina. Yield $88 \%$, white solid, mp 234-236 ${ }^{\circ} \mathrm{C}$ (from ethanol). ${ }^{1} \mathrm{H}$ NMR (DMSO-d $): \delta 2.31(\mathrm{~s}, 6 \mathrm{H}), 3.59(\mathrm{q}, J=5.9 \mathrm{~Hz}, 2 \mathrm{H}$ ), $4.10(\mathrm{t}, J=6.2 \mathrm{~Hz}, 2 \mathrm{H}), 5.98-5.99(\mathrm{~m}, 2 \mathrm{H}), 6.86-6.87(\mathrm{~m}, 2 \mathrm{H}), 7.26(\mathrm{~s}, 1 \mathrm{H}), 7.31-7.39(\mathrm{~m}, 2 \mathrm{H}), 7.66$ $(\mathrm{s}, 2 \mathrm{H}), 9.25$ (br s, $1 \mathrm{H}$, disappeared on treatment with $\left.\mathrm{D}_{2} \mathrm{O}\right), 13.21 \mathrm{ppm}(\mathrm{br} \mathrm{s}, 1 \mathrm{H}$, disappeared on treatment with $\left.\mathrm{D}_{2} \mathrm{O}\right)$. IR: $v$ 1643, $3261 \mathrm{~cm}^{-1}$. Anal. $\left(\mathrm{C}_{23} \mathrm{H}_{21} \mathrm{ClFN}_{3} \mathrm{O}_{3} \mathrm{~S}(473.95)\right) \mathrm{C}, \mathrm{H}, \mathrm{Cl}, \mathrm{F}, \mathrm{N}, \mathrm{S}$.

5-Chloro-3-[(3,5-dimethylphenyl)sulfonyl]-4-fluoro- $N$-(2-phenoxyethyl)-1H-indole-2-

carboxamide (47). Was synthesized as 27 using 2-phenoxyethylamine. Yield 76\%, white solid, mp $198-201{ }^{\circ} \mathrm{C}$ (from ethanol). ${ }^{1} \mathrm{H}$ NMR (DMSO-d $): \delta 2.29(\mathrm{~s}, 6 \mathrm{H}), 3.71(\mathrm{q}, J=5.2 \mathrm{~Hz}, 2 \mathrm{H}), 5.15(\mathrm{t}, J=$ $5.1 \mathrm{~Hz}, 2 \mathrm{H}), 6.93-6.98(\mathrm{~m}, 3 \mathrm{H}), 7.34-7.37(\mathrm{~m}, 5 \mathrm{H}), 7.65(\mathrm{~s}, 2 \mathrm{H}), 9.33$ (br s, 1H, disappeared on treatment with $\left.\mathrm{D}_{2} \mathrm{O}\right), 13.22 \mathrm{ppm}\left(\mathrm{br} \mathrm{s}, 1 \mathrm{H}\right.$, disappeared on treatment with $\mathrm{D}_{2} \mathrm{O}$ ). IR: $v 1643,3204 \mathrm{~cm}^{-}$ 1. Anal. $\left(\mathrm{C}_{25} \mathrm{H}_{22} \mathrm{ClFN}_{2} \mathrm{O}_{4} \mathrm{~S}(500.97)\right) \mathrm{C}, \mathrm{H}, \mathrm{Cl}, \mathrm{F}, \mathrm{N}, \mathrm{S}$. 


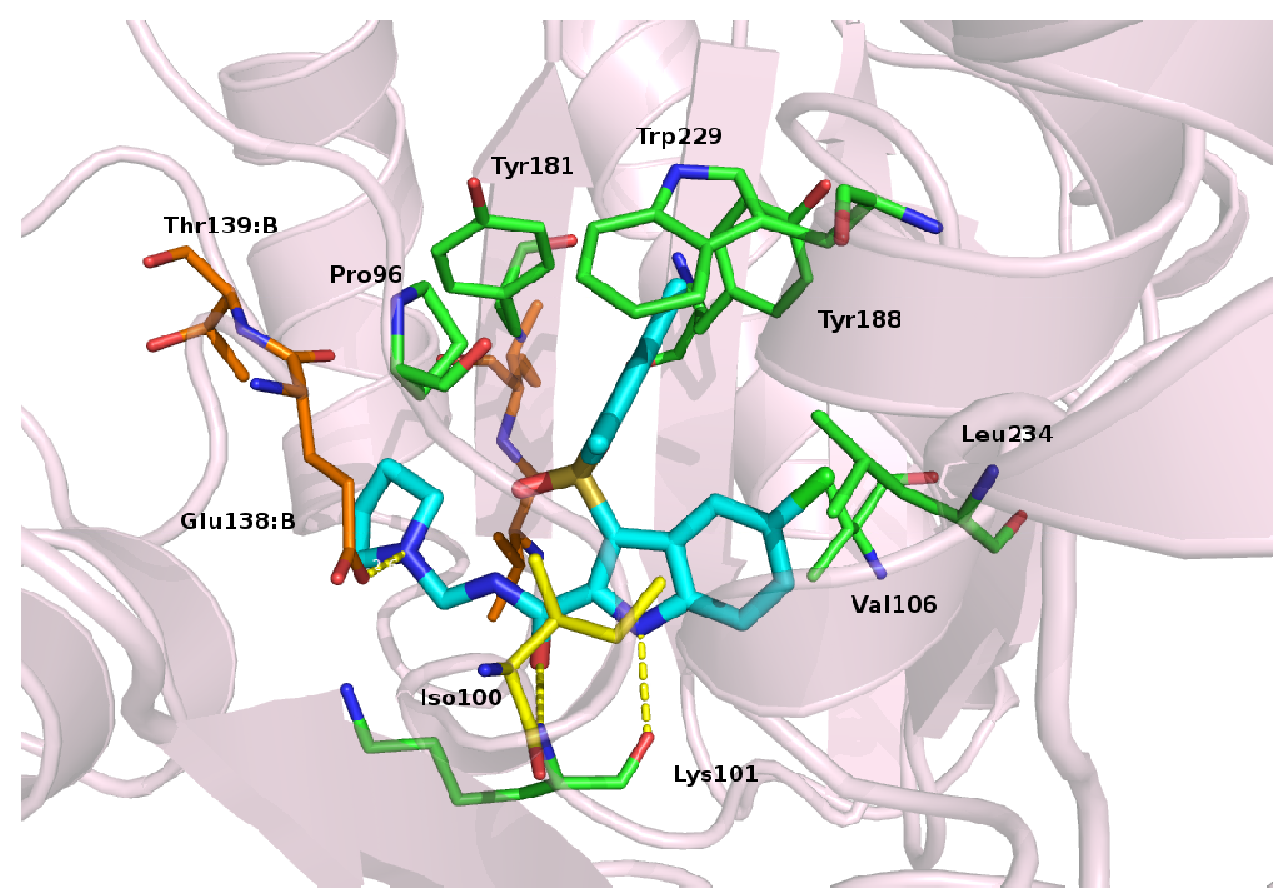

Figure 1S. Binding conformation of derivative 12 (cyan) into the NNBS of the HIV-1 L100I mutated RT. Mutated residues are highlighted as yellow stick. Hydrogen atoms are not shown for clarity. H-bonds are reported as dotted yellow lines. 


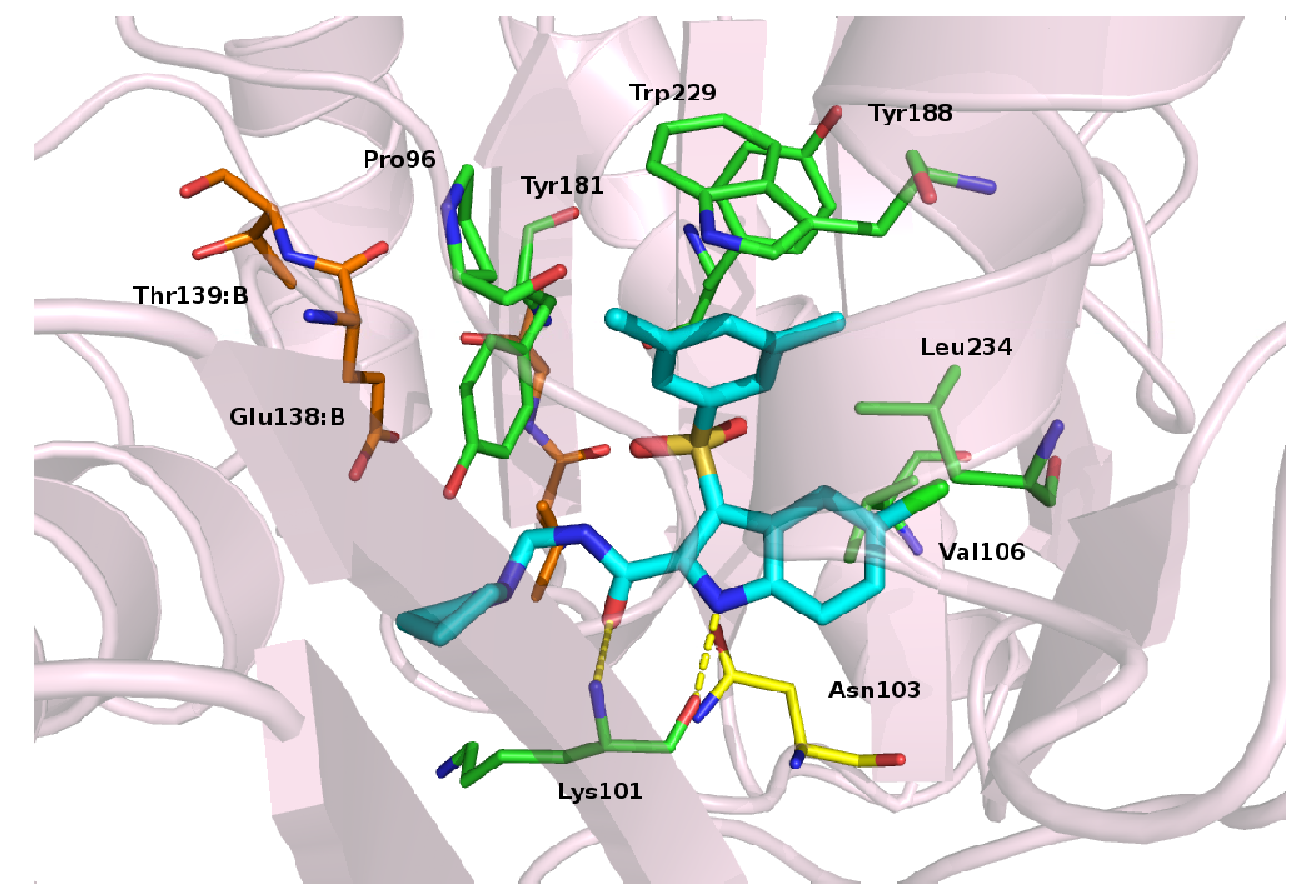

Figure 2S. Binding conformation of derivative 12 (cyan) into the NNBS of the HIV-1 K103N mutated RT. Mutated residues are highlighted as yellow stick. Hydrogen atoms are not shown for clarity. H-bonds are reported as dotted yellow lines. 


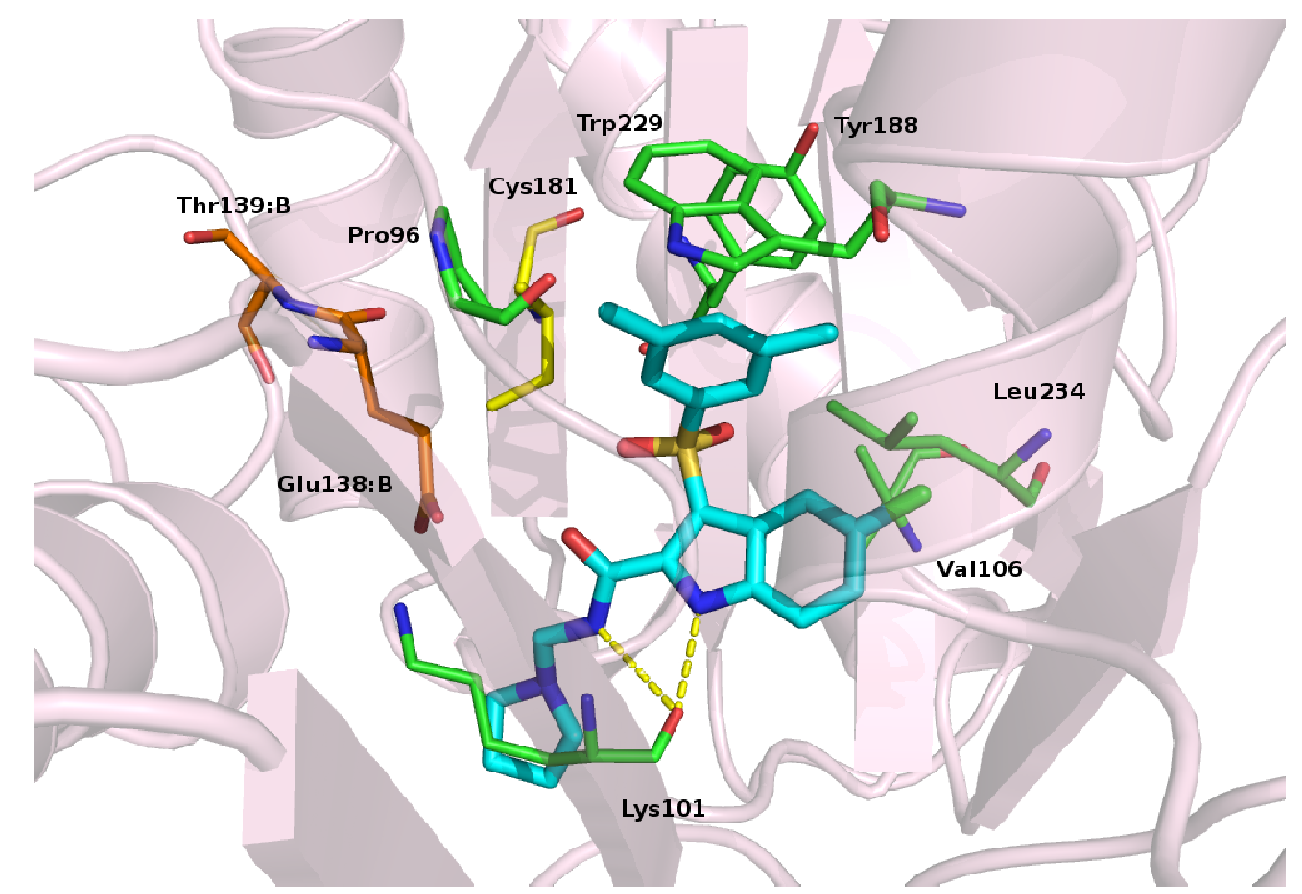

Figure 3S. Binding conformation of derivative 12 (cyan) into the NNBS of the HIV-1 Y181C mutated RT. Mutated residues are highlighted as yellow stick. Hydrogen atoms are not shown for clarity. H-bonds are reported as dotted yellow lines. 
Table 1S. Anti-HIV-1 Activity, Cytotoxicity, Selectivity Index and Relative Factor of Compounds 20, 24-26, 28, 36, 40 and 44 against Mutant HIV-1 Strains in MT-4 Cells.

\begin{tabular}{|c|c|c|c|c|c|}
\hline Compd & HIV-1 Strain & $\mathrm{IC}_{50}(\mathrm{nM})$ & $\mathrm{CC}_{50}(\mathrm{nM})$ & SI & $\mathrm{RF}$ \\
\hline \multirow[t]{6}{*}{20} & $\mathrm{III}_{\mathrm{B}}$ & $3.2 \pm 0.8$ & $>1000$ & $>312$ & 1.0 \\
\hline & L100I & $8.0 \pm 5.6$ & $>1000$ & $>125$ & 2.5 \\
\hline & K103N & $11 \pm 3$ & $>1000$ & $>90$ & 3.5 \\
\hline & Y181C & $23 \pm 6$ & $>1000$ & $>43$ & 7.1 \\
\hline & Y188L & $440 \pm 60$ & $>1000$ & $>2$ & 137.5 \\
\hline & E138K & $14 \pm 0$ & $>1000$ & $>71$ & 4.2 \\
\hline \multirow[t]{6}{*}{24} & $\mathrm{III}_{\mathrm{B}}$ & $11 \pm 7$ & $>1000$ & $>90$ & 1.0 \\
\hline & L100I & $6.1 \pm 2.4$ & $>1000$ & $>164$ & 0.5 \\
\hline & K103N & $11 \pm 0$ & $>1000$ & $>93$ & 1.0 \\
\hline & Y181C & $46 \pm 11$ & $>1000$ & $>21$ & 4.0 \\
\hline & Y188L & $480 \pm 90$ & $>1000$ & $>2$ & 43.6 \\
\hline & E138K & $18 \pm 5$ & $>1000$ & $>55$ & 1.6 \\
\hline \multirow[t]{6}{*}{25} & $\mathrm{III}_{\mathrm{B}}$ & $16 \pm 3$ & $>1000$ & $>62$ & 1.0 \\
\hline & L100I & $11 \pm 8$ & $>1000$ & $>90$ & 0.7 \\
\hline & $\mathrm{K} 103 \mathrm{~N}$ & $15 \pm 3$ & $>1000$ & $>66$ & 0.9 \\
\hline & Y181C & $59 \pm 37$ & $>1000$ & $>17$ & 3.7 \\
\hline & Y188L & $\geq 450$ & $>1000$ & - & - \\
\hline & E138K & $\overline{5} 1 \pm 41$ & $>1000$ & $>19$ & 3.2 \\
\hline \multirow[t]{6}{*}{26} & $\mathrm{III}_{\mathrm{B}}$ & $10 \pm 7$ & $>1000$ & $>100$ & 1.0 \\
\hline & L100I & $7 \pm 5.1$ & $>1000$ & $>142$ & 0.7 \\
\hline & K103N & $11 \pm 5$ & $>1000$ & $>90$ & 1.1 \\
\hline & Y181C & $54 \pm 30$ & $>1000$ & $>18$ & 5.4 \\
\hline & Y188L & $470 \pm 200$ & $>1000$ & $>2$ & 47.0 \\
\hline & E138K & $16 \pm 0$ & $>1000$ & $>62$ & 1.6 \\
\hline \multirow[t]{6}{*}{28} & $\mathrm{III}_{\mathrm{B}}$ & $19 \pm 1$ & $>1000$ & $>52$ & 1.0 \\
\hline & L100I & $84 \pm 8$ & $>1000$ & $>11$ & 4.4 \\
\hline & $\mathrm{K} 103 \mathrm{~N}$ & $80 \pm 5$ & $>1000$ & $>12$ & 4.2 \\
\hline & Y181C & $380 \pm 20$ & $>1000$ & $>2.6$ & 20.0 \\
\hline & Y188L & $>1000$ & $>1000$ & - & - \\
\hline & E138K & $260 \pm 100$ & $>1000$ & $>3.8$ & 13.7 \\
\hline \multirow[t]{6}{*}{36} & $\mathrm{III}_{\mathrm{B}}$ & $21 \pm 3$ & $>1000$ & $>47$ & 1.0 \\
\hline & L100I & $19 \pm 1$ & $>1000$ & $>52$ & 0.9 \\
\hline & $\mathrm{K} 103 \mathrm{~N}$ & $52 \pm 6$ & $>1000$ & $>19$ & 2.5 \\
\hline & Y181C & $100 \pm 10$ & $>1000$ & $>10$ & 4.8 \\
\hline & Y188L & $>1000$ & $>1000$ & - & - \\
\hline & E138K & $65 \pm 3$ & $>1000$ & $>15$ & 3.1 \\
\hline \multirow[t]{6}{*}{40} & $\mathrm{III}_{\mathrm{B}}$ & $15 \pm 1$ & $>1000$ & $>66$ & 1.0 \\
\hline & L100I & $11 \pm 1$ & $>1000$ & $>90$ & 0.7 \\
\hline & K103N & $15 \pm 0$ & $>1000$ & $>66$ & 1.0 \\
\hline & Y181C & $67 \pm 22$ & $>1000$ & $>15$ & 4.4 \\
\hline & Y188L & $>1000$ & $>1000$ & - & - \\
\hline & E138K & $18 \pm 1$ & $>1000$ & $>55$ & 1.2 \\
\hline \multirow[t]{6}{*}{44} & $\mathrm{III}_{\mathrm{B}}$ & $300 \pm 140$ & $>1000$ & $>3.3$ & 1.0 \\
\hline & L100I & $440 \pm 200$ & $>1000$ & $>2.3$ & 1.5 \\
\hline & K103N & $>1000$ & $>1000$ & - & - \\
\hline & Y181C & $>1000$ & $>1000$ & - & - \\
\hline & Y188L & $>1000$ & $>1000$ & - & - \\
\hline & E138K & $380 \pm 20$ & $>1000$ & $>2.6$ & 1.3 \\
\hline
\end{tabular}


Table 2S. Inhibitory Effects of New IASs 12-47 and Reference Compound $\mathbf{4}$ on the Proliferation of Murine Leukemia Cells (L1210) and Human TLymphocyte Cells (Molt4/C8).

\begin{tabular}{|c|c|c|}
\hline \multirow[b]{2}{*}{ Compd } & \multicolumn{2}{|c|}{$\mathrm{CC}_{50}(\mu \mathrm{M})$} \\
\hline & L1210 & Molt4/C8 \\
\hline 12 & $18 \pm 14$ & $4.9 \pm 3.6$ \\
\hline 13 & $11 \pm 2$ & $2.5 \pm 0.1$ \\
\hline 14 & $23 \pm 1$ & $8.6 \pm 2.8$ \\
\hline 15 & $9.0 \pm 1.3$ & $6.5 \pm 3.2$ \\
\hline 16 & $13 \pm 2$ & $6.4 \pm 1.2$ \\
\hline 17 & $11 \pm 0$ & $7.2 \pm 1.2$ \\
\hline 18 & $35 \pm 5$ & $10 \pm 1$ \\
\hline 19 & $43 \pm 11$ & $26 \pm 3$ \\
\hline 20 & $18 \pm 7$ & $6.3 \pm 3.6$ \\
\hline 21 & $43 \pm 1$ & 18 v 12 \\
\hline 22 & $49 \pm 1$ & $42 \pm 8$ \\
\hline 23 & $59 \pm 10$ & $45 \pm 5$ \\
\hline 24 & $\geq 500$ & $306 \pm 12$ \\
\hline 25 & $104 \pm 70$ & $158 \pm 2$ \\
\hline 26 & $12 \pm 6$ & $3.7 \pm 2.2$ \\
\hline 27 & $139 \pm 31$ & $15 \pm 5$ \\
\hline 28 & $5.3 \pm 2.6$ & $6.4 \pm 4.0$ \\
\hline 29 & $3.2 \pm 2.1$ & $4.5 \pm 3.5$ \\
\hline 30 & $14 \pm 0$ & $9.5 \pm 1.9$ \\
\hline 31 & $4.8 \pm 0.1$ & $3.9 \pm 2.7$ \\
\hline 32 & $181 \pm 62$ & $4.4 \pm 2.9$ \\
\hline 33 & $>500$ & $238 \pm 49$ \\
\hline 34 & $59 \pm 20$ & $12 \pm 3$ \\
\hline 35 & $13 \pm 2$ & $8.4 \pm 0.6$ \\
\hline 36 & $215 \pm 63$ & $243 \pm 81$ \\
\hline 37 & $>500$ & $238 \pm 49$ \\
\hline 38 & $>500$ & $\geq 500$ \\
\hline 39 & $42 \pm 9$ & $11 \pm 3$ \\
\hline 40 & $93 \pm 33$ & $66 \pm 17$ \\
\hline 41 & $114 \pm 45$ & $150 \pm 11$ \\
\hline 42 & $192 \pm 13$ & $194 \pm 52$ \\
\hline 43 & $8.9 \pm 1.5$ & $8.5 \pm 0.8$ \\
\hline 44 & $200 \pm 77$ & $231 \pm 96$ \\
\hline 45 & $>500$ & $\geq 500$ \\
\hline 46 & $>500$ & $>500$ \\
\hline 47 & $18 \pm 9$ & $9.0 \pm 1.1$ \\
\hline 4 & $12 \pm 1$ & $3.8 \pm 1.3$ \\
\hline
\end{tabular}


Table 3S. Elemental Analyses of Derivatives 12-47 and 49.

\begin{tabular}{|c|c|c|}
\hline Compd & Calculated & Found \\
\hline 12 & C, 59.25; H, 5.42; Cl, 7.95; N, 9.42; S, 7.19 & C, 59.17; H, 5.38; Cl, 7.80; N, 9.35; S, 7.12 \\
\hline 13 & C, 53.88; H, 4.93; Br, 16.29; N, 8.57; S, 6.54 & C, 53.66; H, 4.90; Br, $16.11 ; \mathrm{N}, 8.30 ; \mathrm{S}, 6.22$ \\
\hline 14 & $\mathrm{C}, 57.88 ; \mathrm{H}, 5.30 ; \mathrm{N}, 12.27 ; \mathrm{S}, 7.02$ & C, 57.59; H, 5.27; N, 12.09; S, 6.89 \\
\hline 15 & C, 56.95; H, 5.00; Cl, 7.64; F, 4.09; N, 9.06; S, 6.91 & $\mathrm{C}, 56.76 ; \mathrm{H}, 4.97 ; \mathrm{Cl}, 7.49 ; \mathrm{F}, 3.93 ; \mathrm{N}, 9.88 ; \mathrm{S}, 6.76$ \\
\hline 16 & $\mathrm{C}, 60.05 ; \mathrm{H}, 5.70 ; \mathrm{Cl}, 7.71 ; \mathrm{N}, 9.14 ; \mathrm{S}, 6.97$ & $\mathrm{C}, 59.88 ; \mathrm{H}, 5.67 ; \mathrm{Cl}, 7.56 ; \mathrm{N}, 9.01 ; \mathrm{S}, 6.84$ \\
\hline 17 & $\mathrm{C}, 54.76 ; \mathrm{H}, 5.20 ; \mathrm{Br}, 15.84 ; \mathrm{N}, 8.33 ; \mathrm{S}, 6.36$ & C, 54.59; H, 5.18; Br, $15.69 ; \mathrm{N}, 8.19 ; \mathrm{S}, 6.21$ \\
\hline 18 & $\mathrm{C}, 58.71 ; \mathrm{H}, 5.57 ; \mathrm{N}, 11.91 ; \mathrm{S}, 6.81$ & $\mathrm{C}, 58.44 ; \mathrm{H}, 5.53 ; \mathrm{N}, 11.74 ; \mathrm{S}, 6.74$ \\
\hline 19 & $\mathrm{C}, 57.79 ; \mathrm{H}, 5.27 ; \mathrm{Cl}, 7.42 ; \mathrm{F}, 3.97 ; \mathrm{N}, 8.79 ; \mathrm{S}, 6.71$ & $\mathrm{C}, 57.66 ; \mathrm{H}, 5.22 ; \mathrm{Cl}, 7.27 ; \mathrm{F}, 3.84 ; \mathrm{N}, 8.52 ; \mathrm{S}, 6.48$ \\
\hline 20 & $\mathrm{C}, 57.20 ; \mathrm{H}, 5.24 ; \mathrm{Cl}, 7.67 ; \mathrm{N}, 9.10 ; \mathrm{S}, 6.94$ & $\mathrm{C}, 56.97 ; \mathrm{H}, 5.22 ; \mathrm{Cl}, 7.52 ; \mathrm{N}, 9.04 ; \mathrm{S}, 6.88$ \\
\hline 21 & $\mathrm{C}, 52.18 ; \mathrm{H}, 4.78 ; \mathrm{Br}, 15.78 ; \mathrm{N}, 8.30 ; \mathrm{S}, 6.33$ & C, 51.89; H, 4.72; Br, 15.59; N, 8.14; S, 6.19 \\
\hline 22 & $\mathrm{C}, 63.64 ; \mathrm{H}, 4.67 ; \mathrm{Cl}, 7.83 ; \mathrm{N}, 6.18 ; \mathrm{S}, 7.08$ & C, 63.48; H, 4.63; Cl, 7.68; N, 6.03; S, 6.92 \\
\hline 23 & $\mathrm{C}, 57.95 ; \mathrm{H}, 4.26 ; \mathrm{Br}, 16.06 ; \mathrm{N}, 5.63 ; \mathrm{S}, 6.45$ & $\mathrm{C}, 57.78 ; \mathrm{H}, 4.21 ; \mathrm{Br}, 15.88 ; \mathrm{N}, 5.50 ; \mathrm{S}, 6.30$ \\
\hline 24 & $\mathrm{C}, 62.19 ; \mathrm{H}, 4.57 ; \mathrm{N}, 9.07 ; \mathrm{S}, 6.92$ & $\mathrm{C}, 61.89 ; \mathrm{H}, 4.52 ; \mathrm{N}, 8.93 ; \mathrm{S}, 6.79$ \\
\hline 25 & C, 61.21; H, 4.28; Cl, 7.53; F, 4.03; N, 5.95; S, 6.81 & $\mathrm{C}, 60.98 ; \mathrm{H}, 4.23 ; \mathrm{Cl}, 7.09 ; \mathrm{F}, 3.81 ; \mathrm{N}, 5.71 ; \mathrm{S}, 6.66$ \\
\hline 26 & C, 55.92; H, 5.12; N, 11.86; S, 6.79 & $\mathrm{C}, 55.66 ; \mathrm{H}, 5.07 ; \mathrm{N}, 11.77 ; \mathrm{S}, 6.58$ \\
\hline 27 & $\mathrm{C}, 55.05 ; \mathrm{H}, 4.83 ; \mathrm{Cl}, 7.39 ; \mathrm{F}, 3.96 ; \mathrm{N}, 8.76 ; \mathrm{S}, 6.68$ & $\mathrm{C}, 54.76 ; \mathrm{H}, 4.80 ; \mathrm{Cl}, 7.26 ; \mathrm{F}, 3.90 ; \mathrm{N}, 8.62 ; \mathrm{S}, 6.45$ \\
\hline 28 & $\mathrm{C}, 60.05 ; \mathrm{H}, 5.70 ; \mathrm{Cl}, 7.71 ; \mathrm{N}, 9.14 ; \mathrm{S}, 6.97$ & $\mathrm{C}, 59.68 ; \mathrm{H}, 5.68 ; \mathrm{Cl}, 7.57 ; \mathrm{N}, 9.02 ; \mathrm{S}, 6.62$ \\
\hline 29 & $\mathrm{C}, 54.76 ; \mathrm{H}, 5.20 ; \mathrm{Br}, 15.84 ; \mathrm{N}, 8.33 ; \mathrm{S}, 6.36$ & C, 54.58; H, 5.18; Br, 15.60; N, 8.19; S, 6.01 \\
\hline 30 & $\mathrm{C}, 58.71 ; \mathrm{H}, 5.57 ; \mathrm{N}, 11.91 ; \mathrm{S}, 6.81$ & $\mathrm{C}, 58.47 ; \mathrm{H}, 5.52 ; \mathrm{N}, 11.84 ; \mathrm{S}, 6.67$ \\
\hline 31 & C, 57.79; H, 5.27; Cl, 7.42; F, 3.97; N, 8.79; S, 6.71 & C, $57.41 ; \mathrm{H}, 5.21 ; \mathrm{Cl}, 7.28 ; \mathrm{F}, 3.84 ; \mathrm{N}, 8.82 ; \mathrm{S}, 6.56$ \\
\hline 32 & $\mathrm{C}, 58.04 ; \mathrm{H}, 5.51 ; \mathrm{Cl}, 7.45 ; \mathrm{N}, 8.83 ; \mathrm{S}, 6.74$ & $\mathrm{C}, 57.89 ; \mathrm{H}, 5.48 ; \mathrm{Cl}, 7.32 ; \mathrm{N}, 8.68 ; \mathrm{S}, 6.59$ \\
\hline 33 & C, $53.08 ; \mathrm{H}, 5.04 ; \mathrm{Br}, 15.35 ; \mathrm{N}, 8.07 ; \mathrm{S}, 6.16$ & C, $52.87 ; \mathrm{H}, 4.95 ; \mathrm{Br}, 15.17 ; \mathrm{N}, 7.92 ; \mathrm{S}, 6.04$ \\
\hline 34 & C, $56.78 ; \mathrm{H}, 5.39 ; \mathrm{N}, 11.52 ; \mathrm{S}, 6.59$ & $\mathrm{C}, 56.49 ; \mathrm{H}, 5.31 ; \mathrm{N}, 11.07 ; \mathrm{S}, 6.01$ \\
\hline 35 & C, 55.92; H, 5.10; Cl, 7.18; F, 3.85; N, 8.51; S, 6.49 & C, 55.77; H, 5.03; Cl, 7.05; F, 3.74; N, 8.38; S, 6.26 \\
\hline 36 & $\mathrm{C}, 64.30 ; \mathrm{H}, 4.96 ; \mathrm{Cl}, 7.59 ; \mathrm{N}, 6.00 ; \mathrm{S}, 6.87$ & $\mathrm{C}, 64.18 ; \mathrm{H}, 4.93 ; \mathrm{Cl}, 7.43 ; \mathrm{N}, 5.89 ; \mathrm{S}, 6.63$ \\
\hline 37 & $\mathrm{C}, 58.71 ; \mathrm{H}, 4.53 ; \mathrm{Br}, 15.62 ; \mathrm{N}, 5.48 ; \mathrm{S}, 6.27$ & C, $58.58 ; \mathrm{H}, 4.51 ; \mathrm{Br}, 15.47 ; \mathrm{N}, 5.40 ; \mathrm{S}, 6.14$ \\
\hline 38 & $\mathrm{C}, 62.88 ; \mathrm{H}, 4.85 ; \mathrm{N}, 8.80 ; \mathrm{S}, 6.71$ & $\mathrm{C}, 62.65 ; \mathrm{H}, 4.80 ; \mathrm{N}, 8.65 ; \mathrm{S}, 6.59$ \\
\hline 39 & $\mathrm{C}, 61.91 ; \mathrm{H}, 4.57 ; \mathrm{Cl}, 7.31 ; \mathrm{F}, 3.92 ; \mathrm{N}, 5.78 ; \mathrm{S}, 6.61$ & $\mathrm{C}, 61.79 ; \mathrm{H}, 4.51 ; \mathrm{Cl}, 7.18 ; \mathrm{F}, 3.78 ; \mathrm{N}, 5.56 ; \mathrm{S}, 6.35$ \\
\hline 40 & $\mathrm{C}, 60.59 ; \mathrm{H}, 4.86 ; \mathrm{Cl}, 7.78 ; \mathrm{N}, 9.22 ; \mathrm{S}, 7.03$ & $\mathrm{C}, 60.36 ; \mathrm{H}, 4.81 ; \mathrm{Cl}, 7.62 ; \mathrm{N}, 9.05 ; \mathrm{S}, 6.88$ \\
\hline 41 & C, 55.20; H, 4.43; Br, 15.97; N, 8.40; S, 6.41 & $\mathrm{C}, 55.01 ; \mathrm{H}, 4.39 ; \mathrm{Br}, 15.82 ; \mathrm{N}, 8.33 ; \mathrm{S}, 6.27$ \\
\hline 42 & C, 59.22; H, 4.75; N, 12.01; S, 6.87 & $\mathrm{C}, 58.98 ; \mathrm{H}, 4.70 ; \mathrm{N}, 11.86 ; \mathrm{S}, 6.64$ \\
\hline 43 & C, 58.29; H, 4.47; Cl, 7.48; F, 4.01; N, 8.87; S, 6.77 & C, 58.02; H, 4.20; Cl, 7.22; F, 3.79; N, 8.23; S, 6.53 \\
\hline 44 & $\mathrm{C}, 62.17 ; \mathrm{H}, 4.80 ; \mathrm{Cl}, 7.34 ; \mathrm{N}, 5.80 ; \mathrm{S}, 6.64$ & $\mathrm{C}, 61.88 ; \mathrm{H}, 4.74 ; \mathrm{Cl}, 7.21 ; \mathrm{N}, 5.57 ; \mathrm{S}, 6.42$ \\
\hline 45 & C, $56.93 ; \mathrm{H}, 4.40 ; \mathrm{Br}, 15.15 ; \mathrm{N}, 5.31 ; \mathrm{S}, 6.08$ & $\mathrm{C}, 56.62 ; \mathrm{H}, 4.32 ; \mathrm{Br}, 15.01 ; \mathrm{N}, 5.18 ; \mathrm{S}, 5.85$ \\
\hline 46 & $\mathrm{C}, 60.84 ; \mathrm{H}, 4.70 ; \mathrm{N}, 8.51 ; \mathrm{S}, 6.50$ & $\mathrm{C}, 60.57 ; \mathrm{H}, 4.68 ; \mathrm{N}, 8.37 ; \mathrm{S}, 6.38$ \\
\hline 47 & C, 59.94; H, 4.43; Cl, 7.08; F, 3.79; N, 5.59; S, 6.40 & $\mathrm{C}, 59.65 ; \mathrm{H}, 4.42 ; \mathrm{Cl}, 6.82 ; \mathrm{F}, 3.58 ; \mathrm{N}, 5.35 ; \mathrm{S}, 6.27$ \\
\hline 49 & $\mathrm{C}, 54.39 ; \mathrm{H}, 4.56 ; \mathrm{N}, 11.19 ; \mathrm{S}, 8.54$ & $\mathrm{C}, 54.11 ; \mathrm{H}, 4.48 ; \mathrm{N}, 10.95 ; \mathrm{S}, 8.28$ \\
\hline
\end{tabular}

